\title{
Two-Loop integrals for precision Higgs boson phenomenology
}

\author{
Francesco Moriello* \\ Institut fur theoretische Physik, ETH Zurich, 8093, Zurich, Switzerland \\ Dipartimento di Fisica, Università di Roma Sapienza, 00185, Roma, Italy \\ INFN Sezione di Roma, 00185 Roma, Italy \\ E-mail: fmoriell@phys.ethz.ch
}

\section{Roberto Bonciani}

Dipartimento di Fisica, Università di Roma Sapienza, 00185 Roma, Italy

INFN Sezione di Roma, 00185 Roma, Italy

E-mail: roberto.bonciani@romal.infn.it

\section{Vittorio Del Duca}

Institute for Theoretical Physics, ETH Zurich, 8093 Zurich, Switzerland

INFN Laboratori Nazionali di Frascati, 00044 Frascati (Roma), Italy

E-mail: delducavaitp.phys.ethz.ch,delduca@lnf.infn.it

\section{Hjalte Frellesvig}

Institute of Nuclear and Particle Physics, NCSR Demokritos, Agia Paraskevi, 15310, Greece

E-mail: frellesvig@inp.demokritos.gr

\section{Johannes M. Henn}

PRISMA Cluster of Excellence, Johannes Gutenberg University, 55099 Mainz, Germany

E-mail: henn@uni-mainz.de

\section{Vladimir A. Smirnov}

Skobeltsyn Inst. of Nuclear Physics of Moscow State University, 119991 Moscow, Russia

E-mail: smirnovatheory.sinp.msu.ru

\begin{abstract}
We report on the analytic computation of the planar master integrals relevant for the next-toleading order QCD corrections to the Higgs boson production in association with a jet in proton collisions, with exact top quark mass dependence. As a by product we compute the NLO QCD corrections to the decay width of the Higgs boson in a $\mathrm{Z}$ boson and a photon. The computation is performed with the differential equations method applied to a set of suitably chosen dimensionally regularized integrals.
\end{abstract}

Loops and Legs in Quantum Field Theory

24-29 April 2016

Leipzig, Germany

\footnotetext{
*Speaker.
} 


\section{Introduction}

The discovery at the LHC of a new particle with a mass of around $125 \mathrm{GeV}[1,2]$ has raised the question whether the long sought Higgs boson of the Standard Model has been found. The experiments were able to confirm that the new particle is a boson since it decays in two photons, two $\mathrm{Z}$ bosons and two $\mathrm{W}$ bosons. Nevertheless more data and more precise theoretical predictions are needed to confirm the properties of the recently discovered particle.

The high $p_{T}$ distribution of the Higgs boson through the process $p p \rightarrow H+$ jet has been proven to be very sensitive to modifications of the Higgs-top coupling and to the effective Higgs-gluon coupling induced by new physics effects in many BSM models [3, 4, 5]. Also, the computation of $H+$ jet production at NLO in perturbative QCD is of fundamental importance, being the theoretical uncertainties in this channel among the largest systematic errors of Higgs analysis [6].

While finite top mass effects at NLO agree very well with the infinite top mass limit for small $p_{T}$, they are no longer negligible when $p_{T} \gtrsim 150 \mathrm{GeV}[7,8,9]$. Nevertheless the rate for $H+$ jet is known analytically with exact top mass only at LO, while the NLO rate is known analytically only in the infinite top mass limit [10]. We report on the computation of all the planar master integrals needed to compute the NLO QCD corrections to $H+$ jet production retaining the exact top mass dependence [11]. In Sec. 2 we define the two relevant integral families. In Sec. 3 we present the differential equations method we used to compute the master integrals. Finally, in Sec. 4 we discuss the solution of the differential equations in terms of iterated integrals.

The same set of master integrals is relevant for the computation of the NLO QCD corrections to the decay width of $H \rightarrow Z \gamma$ [12]. Measuring $\Gamma_{H \rightarrow Z \gamma}$ might provide much information on the process underlying the boson decay. Modifications of the Higgs couplings to $Z \gamma$ are expected if the boson has a different origin [13], if it is a composite state [14], and if new scalar colorless particles, leptons, vector bosons coupling to the Higgs are exchanged into the loops [15]. Moreover the decay channel is clean and suitable for precision experiments. The $Z$ boson may decay leptonically - the final state of $H \rightarrow Z \gamma$ is a lepton pair and a photon - or hadronically - the final state is formed by two jets and a photon. However the two-jet plus photon final state is overwhelmed by the QCD background. Conversely, because of the smaller $p p \rightarrow l^{-} l^{+} \gamma$ background, the decay $H \rightarrow Z \gamma \rightarrow l^{-} l^{+} \gamma$ is much cleaner, and allows for a peak effective mass resolution of about 1-3\% [16].

The LO corrections to the decay width have been computed a while ago, but they provide an analytic result with a rather large uncertainty (10\%) [17], while the NLO QCD corrections were known only numerically [18]. We report on the analytic computation of the NLO QCD corrections to the $H \rightarrow Z \gamma$ decay width [12]. The impact of these corrections is discussed in Sec. 5 .

\section{Integral families}

The integrals we are interested in can be parametrized in terms of two integral families. We 


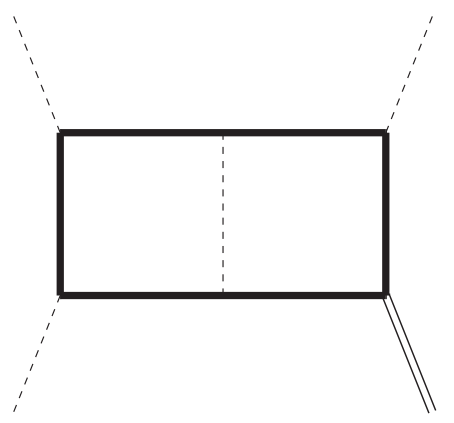

(a)

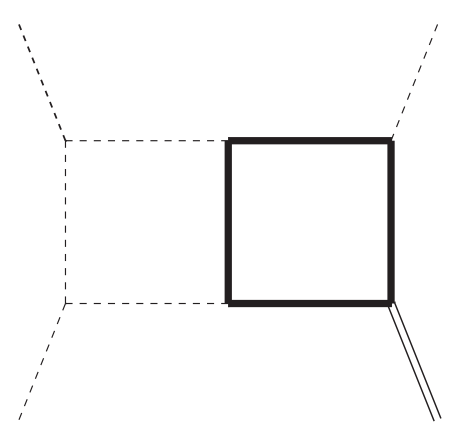

(b)

Figure 1: Representative master integrals of the integral families (a) and (b).

define the following set of inverse propagators for family (a),

$$
\begin{gathered}
d_{1}^{(a)}=m^{2}-k_{1}^{2}, \quad d_{2}^{(a)}=m^{2}-\left(k_{1}+p_{12}\right)^{2}, \quad d_{3}^{(a)}=m^{2}-k_{2}^{2}, \\
d_{4}^{(a)}=m^{2}-\left(k_{2}+p_{12}\right)^{2}, \quad d_{5}^{(a)}=m^{2}-\left(k_{1}+p_{1}\right)^{2}, \quad d_{6}^{(a)}=-\left(k_{1}-k_{2}\right)^{2}, \\
d_{7}^{(a)}=m^{2}-\left(k_{2}-p_{3}\right)^{2}, \quad d_{8}^{(a)}=m^{2}-\left(k_{2}+p_{1}\right)^{2}, \quad d_{9}^{(a)}=m^{2}-\left(k_{1}-p_{3}\right)^{2},
\end{gathered}
$$

and likewise the inverse propagators of family (b),

$$
\begin{gathered}
d_{1}^{(b)}=-k_{1}^{2}, \quad d_{2}^{(b)}=-\left(k_{1}+p_{12}\right)^{2}, \quad d_{3}^{(b)}=m^{2}-k_{2}^{2}, \\
d_{4}^{(b)}=m^{2}-\left(k_{2}+p_{12}\right)^{2}, \quad d_{5}^{(b)}=-\left(k_{1}+p_{1}\right)^{2}, \quad d_{6}^{(b)}=m^{2}-\left(k_{1}-k_{2}\right)^{2}, \\
d_{7}^{(b)}=m^{2}-\left(k_{2}-p_{3}\right)^{2}, \quad d_{8}^{(b)}=m^{2}-\left(k_{2}+p_{1}\right)^{2}, \quad d_{9}^{(b)}=-\left(k_{1}-p_{3}\right)^{2} .
\end{gathered}
$$

The most general integral is defined in $D=4-2 \varepsilon$ space-time dimensions as:

$$
I_{a_{1}, a_{2}, a_{3}, a_{4}, a_{5}, a_{6}, a_{7}, a_{8}, a_{9}}^{(i)}=\int \frac{d^{D} k_{1} d^{D} k_{2}}{i \pi^{D / 2} i \pi^{D / 2}} \frac{\left[d_{8}^{(i)}\right]^{-a_{8}}\left[d_{9}^{(i)}\right]^{-a_{9}}}{\left[d_{1}^{(i)}\right]^{a_{1}}\left[d_{2}^{(i)}\right]^{a_{2}}\left[d_{3}^{(i)}\right]^{a_{3}}\left[d_{4}^{(i)}\right]^{a_{4}}\left[d_{5}^{(i)}\right]^{a_{5}}\left[d_{6}^{(i)}\right]^{a_{6}}\left[d_{7}^{(i)}\right]^{a_{7}}} .
$$

We consider the production of an heavy particle $\mathrm{H}$ of mass $p_{4}^{2}$ via the scattering process,

$$
g\left(p_{1}\right)+g\left(p_{2}\right)+g\left(p_{3}\right) \rightarrow H\left(p_{4}\right),
$$

where $p_{1}^{2}=p_{2}^{2}=p_{3}^{2}=0$. The Mandelstam invariants are

$$
s=\left(p_{1}+p_{2}\right)^{2}, \quad t=\left(p_{1}+p_{3}\right)^{2}, \quad u=\left(p_{2}+p_{3}\right)^{2},
$$

and they are related to the external mass by $s+t+u=p_{4}^{2}$. The physical region corresponds to $s>0, t<0, u<0, p_{4}^{2}>0, m^{2}>0$. The integrals are functions of three invariants, $s, t, p_{4}^{2}$, normalized to the internal mass $m^{2}$.

The full set of integrals is reduced to a minimal set of independent integrals, dubbed master integrals, using the computer program FIRE5 [19]. We compute the master integrals in the euclidean region $s<0, t<0, p_{4}^{2}<0, m^{2}>0$, where no branch cut ambiguities are present. It is then possible to analytically continue the result to the physical region using Feynman prescription, assigning a positive vanishing imaginary part to the external invariants and a negative vanishing imaginary part to the internal masses. 


\section{Canonical form of the differential equations}

In order to analytically compute the master integrals we rely on the differential equations method [20,21, 22] applied to a modified basis of integrals defined to be pure functions of defined weight $[23]^{1}$.

Provided a set of $N$ scalar loop integrals $f$, depending on a set of variables $x$, and working in $D=4-2 \varepsilon$ dimensions, it is possible to define a system of first order differential equations for the integrals, that can be written in total generality as $\left(\partial_{m} \equiv \frac{d}{d x_{m}}\right)$

$$
\partial_{m} f(x, \varepsilon)=A_{m}(x, \varepsilon) f(x, \varepsilon),
$$

where $A_{m}(x, \varepsilon)$ is an $N \times N$ matrix with rational entries of its variables. The matrix $A_{m}(x, \varepsilon)$ satisfies the integrability condition

$$
\partial_{n} A_{m}-\partial_{m} A_{n}+\left[A_{n}, A_{m}\right]=0,
$$

where $\left[A_{n}, A_{m}\right]=A_{n} A_{m}-A_{m} A_{n}$.

The choice of the integral basis is not unique. Performing a linear transformation $B(x, \varepsilon)$ of the integral basis the system of differential equations transforms according to

$$
A \rightarrow B^{-1} \partial_{x} B-B^{-1} A B .
$$

In [23] it was conjectured that with a proper basis choice it is possible to factorize out the $\varepsilon$ dependence of the differential equations:

$$
\partial_{m} f(x, \varepsilon)=\varepsilon A_{m}(x) f(x, \varepsilon) .
$$

Such a system of differential equations is said to be in canonical form. The main virtue of the canonical system of differential equations is that its solution is elementary, and it can be written for general $\varepsilon$ in terms of a path-ordered exponential,

$$
f(x, \varepsilon)=P e^{\varepsilon \int_{C} d A} f(0, \varepsilon),
$$

where $C$ is a path connecting the boundary point to $x$. In practice it is convenient to express the solution as a power series around $\varepsilon=0$. Denoting with $f_{i}(x)$ the coefficient of the order $\varepsilon^{i}$, the different orders of the solution are related by the following recursive relation:

$$
f_{i}(x)=\varepsilon \int_{C} d A(x) f_{i-1} .
$$

The previous relation shows that the solution is expressed to all orders of $\varepsilon$ in terms of Chen iterated integrals [25].

The specific choice of the integral basis leading to the canonical form of the differential equations was achieved using ideas outlined in [23]. It is known that integrals with constant leading singularities [26] satisfy canonical differential equations. Using generalized cuts we look for (combinations of) integrals with simple leading singularities, that can be normalized to unit rescaling

\footnotetext{
${ }^{1}$ Four integrals of family (a) satisfy higher order differential equations, and a canonical basis of integrals in the sense of [23] cannot be found [24]. It is nevertheless possible to obtain an analytic expression in terms of iterated integrals suitable for numeric evaluation [11].
} 
the candidate integrals. This typically leads to a form close to the canonical form. The remaining unwanted terms can be then algorithmically removed from the differential equations shifting the integral basis $[27,28]$.

When the canonical basis is found, the matrix elements of $A(x)$ are $\mathbb{Q}$-linear combinations of logarithms. (The logarithms arguments are known as the letters of the differential equations, while the set of letters is known as the alphabet.) The solution is expressed in terms of pure functions of uniform weight corresponding to the order of the $\varepsilon$ expansion. As we will see in the next section, these considerations will be fundamental for the solution of the differential equations.

\section{Solution of the differential equations}

The function alphabet for the integrals under consideration contains seven independent square roots that cannot be rationalized via a variable change. This means that it is not possible to integrate Eq. (3.6) in terms of Goncharov polylogarithms [29]. However we can find an expression in terms of these functions using the concept of the symbol [29, 30, 31] of an iterated integral. The symbol corresponds to the integration kernels defining the iterated integrals. For the integral basis is chosen to be of uniform weight the symbol of the solution is completely manifest in our differential equations approach:

$$
S\left(f_{i}(x)\right)=\varepsilon S\left(f_{i-1}\right) \otimes A(x) .
$$

The corresponding polylogarithmic functions can be found proceeding in the following algorithmic steps [31,32]. First, one generates a list of function arguments as monomials in the letters appearing in the alphabet. For the classical polylogarithms $\operatorname{Li}_{n}\left(x_{1}\right)$, one requires that $1-x_{1}$ factorizes over the letters appearing in the alphabet. (A caveat is that in principle "spurious" letters might be needed [32].) For $\mathrm{Li}_{2,2}\left(x_{1}, x_{2}\right)$, the condition is that $1-x_{1}, 1-x_{2}, 1-x_{1} x_{2}$ factorize over the alphabet. Second, for each weight $i$, one chooses a maximal set of linearly independent functions for the alphabet (the linear independence can be verified using the symbol). By construction, we can solve the differential equations at every order in terms of this set of functions. Third, we determine the terms in the kernel of the symbol at order $\varepsilon^{i}$ writing the most general ansatz in terms of the lower weight functions, and solving the differential equations at order $\varepsilon^{i}$ for the free coefficients of the ansatz.

When solving the differential equations in terms of the above functions there is a certain freedom in the choice of the set of function arguments. The choice is usually guided by trying to make certain properties of the answer manifest, such as simple branch cut properties. We require the functions to be real valued in the euclidean region $s<0, t<0, p_{4}^{2}<0, m^{2}>0$. This choice results in a faster numeric evaluation.

In practice we find that the alphabet under consideration is quite large so that a reasonably fast computer implementation of the algorithm is challenging. However we use symbol based techniques to reconstruct polylogarithmic functions up to weight two (where the alphabet letters contributing to the result are a relatively small subset of the full alphabet). We then resort to the Chen integral representation of the solution to define a one-fold integral representation of the answer at weight three and four. Parametrizing the integration contour $C$ with $t \in[0,1]$, Eq. (3.6) 
translates to an iterated integral,

$$
f_{i}(x)=\varepsilon \int_{0}^{1} \partial_{t} A(t) f_{i-1}(t) d t
$$

In this language when the weight two functions are known analytically, the weight three functions are one-fold integrals. The weight four functions are two fold integrals of logarithms differentials, and they can be converted to one-fold integrals performing one integration by parts.

The boundary conditions required to fix the solution are determined using the regularity of the pre-canonical integrals and the behavior of the algebraic factors defining the canonical basis in the boundary point. We find convenient to use the boundary point $s=t=p_{4}^{2}=0$. Regularity conditions can be determined using the algorithms of [33]. In the limit all the canonical integrals vanish except the bubble type ones, that can be nevertheless computed algorithmically in terms of Gamma functions.

We have validated the analytic expressions performing numeric checks against the computer program FIESTA3 [34] for randomly selected points of the euclidean region.

\section{Application: phenomenology of the $H \rightarrow Z \gamma$ decay}

In this section we assess the size of the NLO QCD corrections to the decay width of $H \rightarrow Z \gamma$ [12]. We also discuss the relative contributions of top-quark and bottom-quark diagrams.

For reader's convenience we recall the size of the LO corrections. For $m_{H}=125.1 \mathrm{GeV}$, $m_{t}=173.34 \mathrm{GeV}, m_{b}=4.6 \mathrm{GeV}, m_{W}=80.398 \mathrm{GeV}, \Gamma_{W}=2.141 \mathrm{GeV}, m_{Z}=91.1876 \mathrm{GeV}, s_{W}^{2}=$ $0.23149, \alpha=1 / 128, G_{F}=1.16637 \cdot 10^{-5}$, the QCD width at LO is $0.02 \mathrm{KeV}$. In Table 1 , we report the LO corrections of the top-quark diagrams and the bottom-quark diagrams individually, and the top-bottom interference. The QCD width is dominated by the top diagrams, being the bottom contributes only one per mille of the full QCD LO width.

The NLO QCD corrections to the decay width are numerically tiny. In Fig. 2, we plot the decay width as a function of the Higgs mass including the LO and NLO QCD contributions (topquark and bottom-quark). In Fig. 3 we plot the two-loop QCD correction for the top diagrams, the bottom diagrams, and the complete correction. Similarly to the LO the top correction is the dominant one, and interferes destructively with the bottom correction. In Table 3 we report the numeric values of the decay width for $m_{H}=125.1 \mathrm{GeV}$ and $\alpha_{S}\left(m_{H}^{2}\right)=0.115$ : the two-loop QCD corrections amount to $0.22 \%$ of the $\mathrm{LO}$ width, with the top loop adding a $0.3 \%$ and the bottom loop subtracting a $0.08 \%$. The NLO corrections are in full agreement with the independent analytic computation of [35] and the numeric results of [18].

We note that the two-loop QCD contribution is about 75\% of the one-loop QCD contribution. In principle higher QCD orders would be necessary to stabilize the QCD perturbative series. However up to NLO accuracy the QCD contribution is numerically tiny and much smaller than the EW corrections. This is shown in Table 2 where we report the total $\mathrm{LO}$ width $(6.67 \mathrm{KeV})$, the relative contribution of the QCD loop, the $W$ boson loop, and the QCD- $W$ boson interference to the LO width. We see that the QCD corrections contribute only three per mille to the total width at LO. Thus we expect the NLO EW corrections to be more relevant than the QCD ones. 


\begin{tabular}{|c|c|c|c|}
\hline$\Gamma_{Q C D}^{(1 l)}(\mathrm{KeV})$ & $\Gamma_{t}^{(1 l)} / \Gamma_{Q C D}^{(1 l)}$ & $\Gamma_{b}^{(1 l)} / \Gamma_{Q C D}^{(1 l)}$ & $\operatorname{Interf}_{t-b}^{(1 l)} / \Gamma_{Q C D}^{(1 l)}$ \\
\hline 0.02 & 1.052 & $1 \cdot 10^{-3}$ & -0.053 \\
\hline
\end{tabular}

Table 1: Values of the QCD LO width, and of the relative contributions of the top loop, the bottom loop and of the interference between top and bottom loops, at $m_{H}=125.1 \mathrm{GeV}$.

\begin{tabular}{|c|c|c|c|}
\hline$\Gamma^{(1 l)}(\mathrm{KeV})$ & $\Gamma_{Q C D}^{(1 l)} / \Gamma^{(1 l)}$ & $\Gamma_{W}^{(1 l)} / \Gamma^{(1 l)}$ & $\operatorname{Interf}_{Q C D-W}^{(1 l)} / \Gamma^{(1 l)}$ \\
\hline 6.67 & $3 \cdot 10^{-3}$ & 1.112 & -0.115 \\
\hline
\end{tabular}

Table 2: Values of the LO width, and of the relative contributions of the QCD loops, the $W$-boson loop and of the interference between the QCD and the $W$-boson loops, at $m_{H}=125.1 \mathrm{GeV}$.

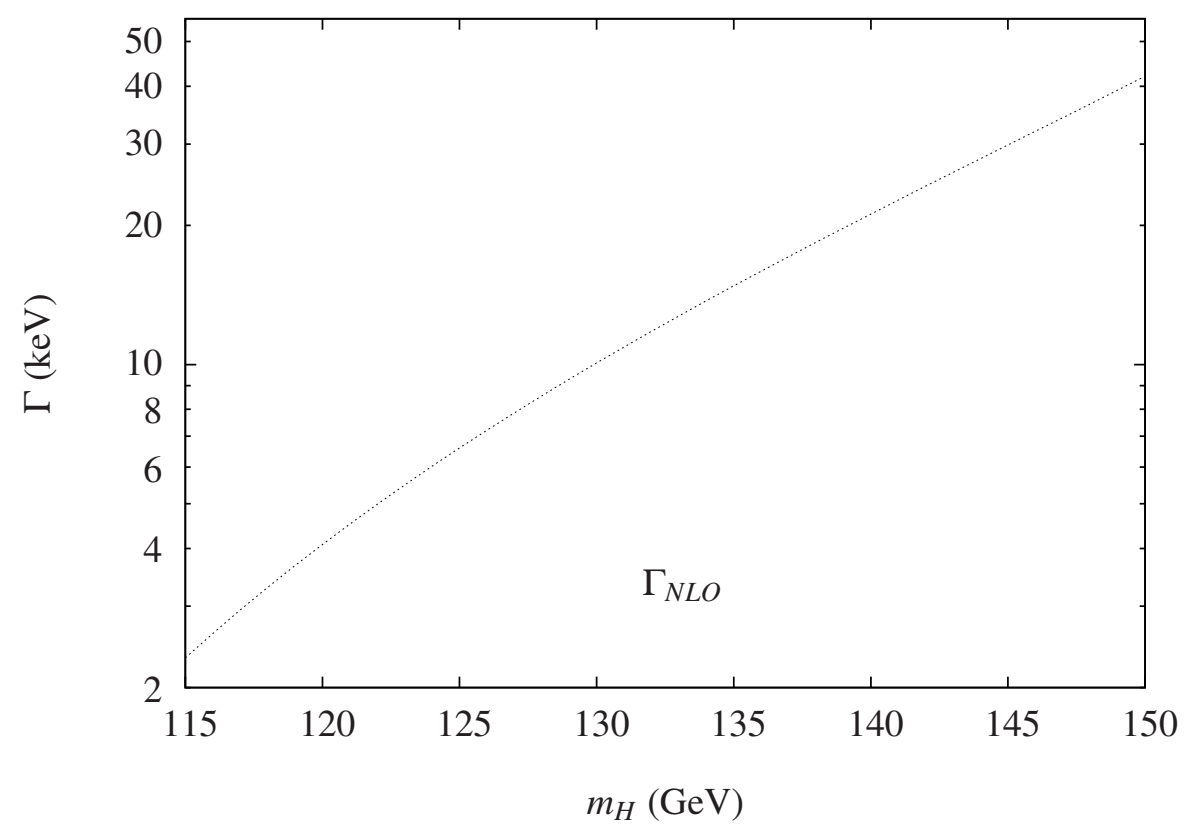

Figure 2: $\Gamma_{H \rightarrow Z \gamma}$ including LO and NLO QCD contributions.

\begin{tabular}{|c|c|c|c|}
\hline$\Gamma(\mathrm{KeV})$ & $\delta_{Q C D}$ (top) & $\delta_{Q C D}$ (bottom) & $\delta_{Q C D}$ \\
\hline 6.68 & $3 \cdot 10^{-3}$ & $-0.8 \cdot 10^{-3}$ & $2.2 \cdot 10^{-3}$ \\
\hline
\end{tabular}

Table 3: Values of the NLO QCD width, and of the NLO QCD corrections $\delta_{Q C D}$ with respect to the leading order contribution, at $m_{H}=125.1 \mathrm{GeV}$. 


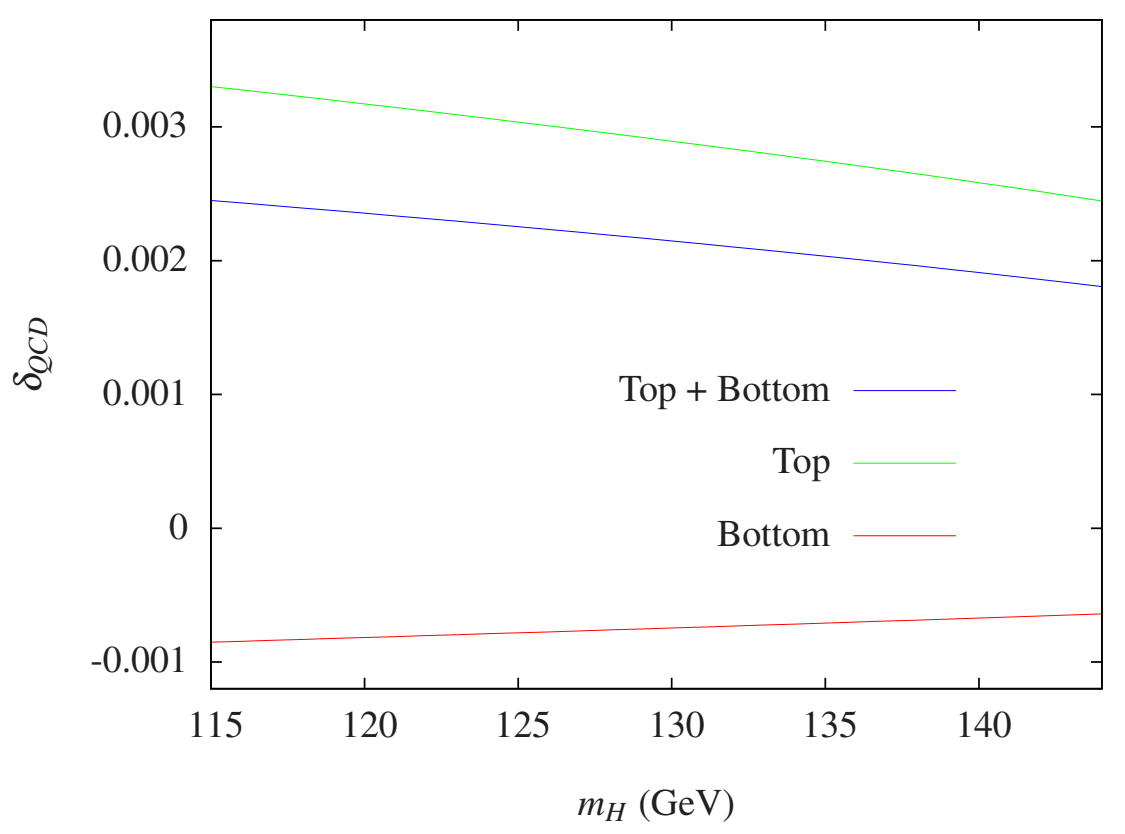

Figure 3: The relative size of the NLO QCD corrections, $\delta_{Q C D}$, to the decay width with respect to the leading order contribution. In this figure we plot the contribution of the top loop only, the bottom loop only, and the top and bottom loops.

\section{Conclusions}

We reported on the analytic computation of all the planar master integrals relevant for the NLO QCD corrections to Higgs plus jet production in proton collisions retaining the full top quark mass dependence. The result is expressed in terms of iterated integrals of simple transcendental functions and it is suitable for numeric evaluations.

\section{References}

[1] G. Aad et al. [ATLAS Collaboration], Phys. Lett. B 716, 1 (2012) [arXiv:1207.7214 [hep-ex]].

[2] S. Chatrchyan et al. [CMS Collaboration], Phys. Lett. B 716, 30 (2012) [arXiv:1207.7235 [hep-ex]].

[3] C. Grojean, E. Salvioni, M. Schlaffer and A. Weiler, JHEP 1405, 022 (2014) [arXiv:1312.3317 [hep-ph]].

[4] A. Azatov and A. Paul, JHEP 1401, 014 (2014) [arXiv:1309.5273 [hep-ph]].

[5] D. Ghosh and M. Wiebusch, Phys. Rev. D 91, no. 3, 031701 (2015) [arXiv:1411.2029 [hep-ph]].

[6] S. Heinemeyer et al. [LHC Higgs Cross Section Working Group Collaboration], arXiv:1307.1347 [hep-ph].

[7] R. V. Harlander, T. Neumann, K. J. Ozeren and M. Wiesemann, JHEP 1208, 139 (2012) [arXiv:1206.0157 [hep-ph]].

[8] R. Frederix, S. Frixione, E. Vryonidou and M. Wiesemann, arXiv:1604.03017 [hep-ph]. 
[9] F. Caola, S. Forte, S. Marzani, C. Muselli and G. Vita, arXiv:1606.04100 [hep-ph].

[10] T. Gehrmann, M. Jaquier, E. W. N. Glover and A. Koukoutsakis, JHEP 1202, 056 (2012) [arXiv:1112.3554 [hep-ph]].

[11] R. Bonciani, V. Del Duca, H. Frellesvig, J. M. Henn, F. Moriello and V. A. Smirnov, In preparation.

[12] R. Bonciani, V. Del Duca, H. Frellesvig, J. M. Henn, F. Moriello and V. A. Smirnov, JHEP 1508, 108 (2015) [arXiv:1505.00567 [hep-ph]].

[13] I. Low, J. Lykken and G. Shaughnessy, Phys. Rev. D 84, 035027 (2011) [arXiv:1105.4587 [hep-ph]].

[14] A. Azatov, R. Contino, A. Di Iura and J. Galloway, Phys. Rev. D 88, no. 7, 075019 (2013) [arXiv:1308.2676 [hep-ph]].

[15] C. W. Chiang and K. Yagyu, Phys. Rev. D 87, no. 3, 033003 (2013) [arXiv:1207.1065 [hep-ph]].

[16] S. Chatrchyan et al. [CMS Collaboration], Phys. Lett. B 726, 587 (2013) [arXiv:1307.5515 [hep-ex]].

[17] R. N. Cahn, M. S. Chanowitz and N. Fleishon, Phys. Lett. B 82, 113 (1979).

[18] M. Spira, A. Djouadi and P. M. Zerwas, Phys. Lett. B 276, 350 (1992).

[19] A. V. Smirnov, Comput. Phys. Commun. 189, 182 (2015) [arXiv:1408.2372 [hep-ph]].

[20] A. V. Kotikov, Phys. Lett. B 254, 158 (1991).

[21] E. Remiddi, Nuovo Cim. A 110, 1435 (1997) [hep-th/9711188].

[22] T. Gehrmann and E. Remiddi, Nucl. Phys. B 580, 485 (2000) [hep-ph/9912329].

[23] J. M. Henn, Phys. Rev. Lett. 110, 251601 (2013) [arXiv:1304.1806 [hep-th]].

[24] J. M. Henn, J. Phys. A 48, 153001 (2015) [arXiv:1412.2296 [hep-ph]].

[25] K. T. Chen, Bull. Am. Math. Soc. 83, 831 (1977).

[26] N. Arkani-Hamed, J. L. Bourjaily, F. Cachazo and J. Trnka, JHEP 1206, 125 (2012) [arXiv:1012.6032 [hep-th]].

[27] S. Caron-Huot and J. M. Henn, JHEP 1406, 114 (2014) [arXiv:1404.2922 [hep-th]].

[28] T. Gehrmann, A. von Manteuffel, L. Tancredi and E. Weihs, JHEP 1406, 032 (2014) [arXiv:1404.4853 [hep-ph]].

[29] A. B. Goncharov, Math. Res. Lett. 5, 497 (1998) [arXiv:1105.2076 [math.AG]].

[30] F. C. S. Brown, Annales Sci. Ecole Norm. Sup. 42, 371 (2009) [arXiv:math/0606419 [math.AG]].

[31] A. B. Goncharov, M. Spradlin, C. Vergu and A. Volovich, Phys. Rev. Lett. 105, 151605 (2010) [arXiv:1006.5703 [hep-th]].

[32] C. Duhr, H. Gangl and J. R. Rhodes, JHEP 1210, 075 (2012) [arXiv:1110.0458 [math-ph]].

[33] V. A. Smirnov, Commun. Math. Phys. 134, 109 (1990).

[34] A. V. Smirnov, Comput. Phys. Commun. 185, 2090 (2014) [arXiv:1312.3186 [hep-ph]].

[35] T. Gehrmann, S. Guns and D. Kara, JHEP 1509, 038 (2015) [arXiv:1505.00561 [hep-ph]]. 